\title{
Theory and Applications of Graphs
}

Volume 6 | Issue 2

Article 3

October 2019

\section{Laplacian Spectral Characterization of Signed Sun Graphs}

Fatemeh Motialah

Shiraz University, fmotialah@shirazu.ac.ir

Mohammad Hassan Shirdareh Haghighi

Shiraz University, shirdareh@shirazu.ac.ir

Follow this and additional works at: https://digitalcommons.georgiasouthern.edu/tag

Part of the Discrete Mathematics and Combinatorics Commons

\section{Recommended Citation}

Motialah, Fatemeh and Shirdareh Haghighi, Mohammad Hassan (2019) "Laplacian Spectral

Characterization of Signed Sun Graphs," Theory and Applications of Graphs: Vol. 6 : Iss. 2 , Article 3.

DOI: $10.20429 / \operatorname{tag} .2019 .060203$

Available at: https://digitalcommons.georgiasouthern.edu/tag/vol6/iss2/3

This article is brought to you for free and open access by the Journals at Digital Commons@Georgia Southern. It has been accepted for inclusion in Theory and Applications of Graphs by an authorized administrator of Digital Commons@Georgia Southern. For more information, please contact digitalcommons@georgiasouthern.edu. 


\section{Laplacian Spectral Characterization of Signed Sun Graphs}

\section{Cover Page Footnote}

The authors would like to thank the anonymous referees for reviewing the paper and giving helpful comments. 


\begin{abstract}
A sun $S G_{n}$ is a graph of order $2 n$ consisting of a cycle $C_{n}, n \geq 3$, to each vertex of it a pendant edge is attached. In this paper, we prove that unbalanced signed sun graphs are determined by their Laplacian spectra. Also we show that a balanced signed sun graph is determined by its Laplacian spectrum if and only if $n$ is odd.
\end{abstract}

\title{
1 Introduction and Preliminaries
}

Spectral characterizations of certain classes of graphs is an important subject in spectral graph theory. This means if a graph $G$ in the specified class is cospectral with a graph $H$ (not necessarily in the class), then $G$ and $H$ are isomorphic. This study is extended to signed graphs by some authors [1].

In this paper we assume that all graphs are simple, i.e. without any loops or multiple edges.

Recall that a signed graph $\Lambda=(G, \sigma)$ is a simple graph $G=(V(G), E(G))$ equipped with a signed function $\sigma: E(G) \rightarrow\{+,-\}$.

The adjacency matrix of a signed graph $\Lambda=(G, \sigma)$ is defined as $A(\Lambda)=\left(a_{i j}^{\sigma}\right)$ with $a_{i j}^{\sigma}=\sigma(i j) a_{i j}$ where $A(G)=\left(a_{i j}\right)$ is the usual adjacency matrix of $G$. Also the Laplacian matrix of $\Lambda$ is defined as $L(\Lambda)=D(G)-A(\Lambda)$.

If all the edge signs are positive then we have the usual definitions of the adjacency and Laplacian matrices. If all the edge signs are negative, then $A(\Lambda)=-A(G)$ and $L(\Lambda)=$ $D(G)+A(G)$, which the latter is called the signless Laplacian matrix of the graph $G$.

It is natural to consider the signed graphs of the classes of graphs that are determined by their spectra and ask if their corresponding signed graphs can be determined by their spectra, too.

The same study can be done for the corresponding Laplacian matrices of signed graphs. For example, the spectral characterization of lollipop graphs is given in [4] and [7] and that of the Laplacian of signed lollipop graphs in [1]. But the situation of signed graphs is slightly different and needs the notion of switching.

For a signed graph $\Lambda=(G, \sigma)$ and $U \subseteq V(G)$, let $\Lambda^{U}$ be the signed graph obtained from $\Lambda$ by reversing the signatures of the edges in the cut $[U, V(G) \backslash U]$. Namely $\sigma_{\Lambda^{U}}(e)=-\sigma_{\Lambda}(e)$ for any edge $e$ between $U$ and $V(G) \backslash U$ and $\sigma_{\Lambda^{U}}(e)=\sigma_{\Lambda}(e)$ otherwise. The signed graph $\Lambda^{U}$ is called a switching of $\Lambda$, and $\Lambda$ and $\Lambda^{U}$ are called switching isomorphic, for the following well-known and easy-to-prove theorem.

Theorem 1.1. The adjacency (Laplacian) matrices of $\Lambda$ and $\Lambda^{U}$ are similar.

Corollary 1.2. The adjacency (Laplacian) matrices of $\Lambda$ and $\Lambda^{U}$ have the same characteristic polynomials.

Definition 1.1. Two signed graphs are said to be A-cospectral (L-cospectral) if they have the same adjacency (Laplacian) characteristic polynomials. Also we say that a signed graph $\Lambda$ is determined by its adjacency (Laplacian) spectrum if every graph that is A-cospectral (L-cospectral) to $\Lambda$ is switching isomorphic to $\Lambda$. 
In this sense, when we say that an unsigned graph is determined by its spectrum or Laplacian spectrum or signless Laplacian spectrum, we mean it is determined up to isomorphism.

Definition 1.2. A sun $S G_{n}$ is a graph of order $2 n$ consisting of a cycle $C_{n}, n \geq 3$, to each vertex of it a pendant edge is attached.

R. Boulet in [3] proves that a sun is determined by its Laplacian spectrum. Also M. Mirzakhan and D. Kiani in [9], show that the sun graphs are determined by their signless Laplacian spectra.

In this paper, we give the Laplacian spectral characterization of signed sun graphs.

Boulet [3] also shows that a sun that its corresponding cycle is odd, is determined by its adjacency spectrum while he provides examples with even cycles that can not be determined by their adjacency spectra ([3, Remark 2]). In [5], Bu et al. studied signless Laplacian spectral characterizations of some generalized sun graphs.

A walk of length $k$ in a signed graph is, as usual, a sequence of vertices $v_{0}, v_{1}, \ldots, v_{k}$ in which $v_{i-1}$ and $v_{i}$, are adjacent, for $i=1,2, \ldots, k$. The sign of a walk is the product the signs of its edges. Thus, a walk is either positive or negative, depending on whether it has an even or odd number of negative edges, respectively. So, the sign of a cycle in a signed graph is positive, if it contains an even number of negative edges, otherwise it is negative. A triangle is thus a walk of length 3 from a vertex to itself.

Definition 1.3. A signed graph is said to be balanced if all of its cycles (if any) are positive, otherwise it is unbalanced.

By $t_{\Lambda}^{+}$(resp., $t_{\Lambda}^{-}$) we mean the number of balanced (resp., unbalanced) triangles in $\Lambda$.

The signatures of the edges of an induced tree is irrelevant in signed graphs up to switching isomorphism. Hence in a signed unicyclic graph $\Lambda=(G, \sigma)$, we have just two non-switching isomorphic structures: either all edges are positive, or the unique cycle has only one negative edge; which can be any of its edges. The former is a balanced graph and the latter is unbalanced. We denote them by $\Lambda=(G,+)$ and $\Lambda=(G,-)$, respectively.

Next we recall a formula useful to compute the coefficients of Laplacian polynomial of signed graphs from [2] and [6].

Definition 1.4. A signed $T U$-subgraph $H$ of a signed graph $\Lambda$ is a spanning subgraph whose components are either trees or unbalanced unicyclic graphs.

If $H$ is a signed TU-subgraph, then we can write $H=\cup_{i=1}^{t} T_{i} \cup_{j=1}^{c} U_{j}$, where if any, the $T_{i}$ 's are trees and the $U_{j}$ 's are unbalanced unicyclic graphs. The weight of the signed TU-subgraph $H$ is defined as $w(H)=4^{c} \prod_{i=1}^{t}\left|T_{i}\right|$.

Theorem 1.3. ([2, Theorem 3.9]) Let $\Lambda$ be a signed graph and $\psi(\Lambda, x)=x^{n}+b_{1} x^{n-1}+$ $\ldots+b_{n-1} x+b_{n}$ be its Laplacian characteristic polynomial. Then

$$
b_{i}=(-1)^{i} \Sigma_{H \in \mathcal{H}_{i}} w(H) \quad i=1,2, \ldots, n,
$$

where $\mathcal{H}_{i}$ denotes the set of signed TU-subgraphs of $\Lambda$ built on $i$ edges. 
Let $S_{k}=\sum_{i=1}^{n} \mu_{i}^{k} \quad(k=0,1,2, \ldots)$ be the $k$-th spectral moment for the Laplacian spectrum of a signed graph $\Lambda$. Let $\Lambda=(G, \sigma)$ be a signed graph with $n$ vertices, $m$ edges and degree sequence $\left(d_{1}, d_{2}, \ldots, d_{n}\right)$.

Lemma 1.4. ([1, Theorem 3.4])

$$
S_{0}=n, \quad S_{1}=\sum_{i=1}^{n} d_{i}=2 m, \quad S_{2}=2 m+\sum_{i=1}^{n} d_{i}^{2}
$$

and

$$
S_{3}=6\left(t^{-}-t^{+}\right)+3 \Sigma_{i=1}^{n} d_{i}^{2}+\sum_{i=1}^{n} d_{i}^{3} .
$$

For an unsigned graph, it is well-known that the multiplicity of 0 as a root of the Laplacian characteristic polynomial counts the number of components, while in a signed graph it counts the number of balanced components.

Theorem 1.5. ([1, Theorem 3.5]) Let $\Lambda=(G, \sigma)$ and $\Gamma=(H, \tau)$ be two L-cospectral signed graphs. Then,

(i) $\Lambda$ and $\Gamma$ have the same number of vertices and edges;

(ii) $\Lambda$ and $\Gamma$ have the same number of balanced components;

(iii) $\Lambda$ and $\Gamma$ have the same Laplacian spectral moments;

(iv) $\Lambda$ and $\Gamma$ have the same sum of squares of degrees, $\sum_{i=1}^{n} d_{G}\left(v_{i}\right)^{2}=\sum_{i=1}^{n} d_{H}\left(v_{i}\right)^{2}$;

(v) $6\left(t_{\Lambda}^{-}-t_{\Lambda}^{+}\right)+\sum_{i=1}^{n} d_{G}\left(v_{i}\right)^{3}=6\left(t_{\Gamma}^{-}-t_{\Gamma}^{+}\right)+\sum_{i=1}^{n} d_{H}\left(v_{i}\right)^{3}$.

The following is the interlacing theorem for signed graphs [1, Theorem 2.5]:

Theorem 1.6. Let $\Lambda=(G, \sigma)$ be a signed graph and e be an edge of $\Lambda$. Then

$$
\mu_{1}(\Lambda) \geq \mu_{1}(\Lambda-e) \geq \ldots \geq \mu_{n}(\Lambda) \geq \mu_{n}(\Lambda-e)
$$

We need also the following well-known lemma, which can be proved plainly.

Lemma 1.7. Let $G$ be a graph with two pendant vertices sharing the same neighbour. Then it has 1 as a Laplacian eigenvalue.

\section{Main Theorem}

A complete analysis of Laplacian-spectra characterization of signed sun graphs is given in this section.

Lemma 2.1. Let $\Lambda=\left(S G_{n}, \sigma\right)$. The eigenvalues of the matrix $L(\Lambda)=\left(\begin{array}{cc}3 I_{n}-A\left(C_{n}\right) & -I_{n} \\ -I_{n} & I_{n}\end{array}\right)$ are $\left(2-\lambda_{i}\right) \pm \sqrt{\left(\lambda_{i}-1\right)^{2}+1}, i=0, \ldots, n-1$; where $\lambda_{i}=\cos \frac{2 i+1}{n} \pi$ if $\sigma=-$, and $\lambda_{i}=\cos \frac{2 i}{n} \pi$ if $\sigma=+$. 
Proof. The proof is very similar to the proof of [9, Lemma 3.1], and is omitted.

Corollary 2.2. If either $\sigma=+$ and $n$ is even, or $\sigma=-$ and $n$ is odd, then $\mu_{1}(\Lambda)=3+\sqrt{5}$ with multiplicity one. Otherwise, $\mu_{1}(\Lambda)<3+\sqrt{5}$.

Although the following Lemma can be deduced from the previous one, we can simply see it by noticing that

$$
I-L(\Lambda)=\left(\begin{array}{cc}
-2 I_{n}+A\left(C_{n}\right) & I_{n} \\
I_{n} & 0
\end{array}\right)
$$

and $\operatorname{det}(I-L(\Lambda)) \neq 0$, since the rows of this matrix are linearly independent. Therefore,

Lemma 2.3. The signed sun graph $\Lambda=\left(S G_{n}, \sigma\right)$ does not have 1 as a Laplacian eigenvalue.

Proposition 2.1. If $\Gamma$ is an $L$-cospectral signed graph with $\Lambda=\left(S G_{n},-\right)$, then $\Gamma$ is a connected unicyclic unbalanced graph.

Proof. Since $\Lambda$ is unbalanced, by Theorem 1.5 (ii), $\Gamma$ has only unbalanced components. Therefore no component of $\Gamma$ is a tree, and by Theorem 1.5 (i), since $\Gamma$ and $\Lambda$ have the same order and size, components of $\Gamma$ are unicyclic. Let $t$ be the number of (unbalanced unicyclic) components of $\Gamma$. So $4^{t}=b_{2 n}(\Gamma)=b_{2 n}(\Lambda)=4$. Consequently $t=1$ and $\Gamma$ is connected.

Lemma 2.4. Let $\Gamma$ be a L-cospectral with $\Lambda=\left(S G_{n}, \sigma\right)$, then $\Delta(\Gamma) \leq 4$.

Proof. From [8, Theorem 3.10] and Corollary 2.2 we have $3+\sqrt{5} \geq \mu_{1} \geq \Delta+1$, and the result follows.

Hereafter, in a specified graph $\Gamma$, let $a_{i}$ denote the number of vertices of degree $i, i=$ $0,1,2, \cdots$. If $\Gamma$ is L-cospectral with $\Lambda=\left(S G_{n}, \sigma\right)$, then by Lemma $2.4, a_{i}=0$ for $i \geq 5$. Also, because of the multiplicity of 0 (Theorem 1.5 (ii)), $a_{0} \leq 1$ and $a_{0}=0$ whenever $\sigma=-$.

Lemma 2.5. For a signed graph $\Gamma$ which is L-cospectral with $\Lambda=\left(S G_{n}, \sigma\right)$, we have:

$$
\begin{gathered}
\sum_{i=0}^{4} a_{i}=2 n, \\
\sum_{i=0}^{4} i a_{i}=4 n, \\
\sum_{i=0}^{4} i^{2} a_{i}=10 n, \\
a_{0}+a_{3}+3 a_{4}=n .
\end{gathered}
$$

Proof. The equations (1), (2) and (3) follow from Lemma 1.4, and together deduce (4).

A part of our main theorem is the special case $n=3$.

Theorem 2.6. Let $\Gamma$ be L-cospectral with $\Lambda=\left(S G_{3}, \sigma\right)$. Then $\Gamma$ is switching isomorphic to $\Lambda$. 
Proof. First suppose that $\sigma=+$. By equation (4), $a_{0}+a_{3}+3 a_{4}=3$, which says that $a_{4} \leq 1$. If $a_{4}=1$, then $S_{5}$, the star of order 5 , is a subgraph of $\Gamma$. We can reach $S_{5}+K_{1}$ by deleting two edges of $\Gamma$. The star $S_{5}$ has 1 as a Laplacian eigenvalue with multiplicity 3. The interlacing Theorem (1.6) implies that 1 is a Laplacian eigenvalue of $\Gamma$, while 1 is not an eigenvalue of $\Lambda=\left(S G_{3},+\right)$, by Lemma 2.3. This contradiction shows that $a_{4}=0$ and hence $a_{0}+a_{3}=3$. We have $a_{0} \leq 1$. Suppose that $a_{0}=1$. Then $a_{3}=2, a_{1}=0$ and $a_{2}=3$, by equations (1), (2) and (3). So $\Gamma=K_{1}+\Omega$ where $\Omega$ is an unbalanced graph whose underlying graph is obtained from $C_{5}$ by adding a chord. By Theorem 1.3, $\left|b_{5}(\Lambda)\right|=18$. Also $b_{5}(\Lambda)=b_{5}(\Gamma)=b_{5}(\Omega)$. We have $b_{5}(\Omega)=-4 \alpha$, where $\alpha$ is the number of unbalanced unicyclic subgraphs of $\Omega$ on 5 edges (these subgraphs are obtained by deleting a possible single edge from $\Omega$ ). So $4 \alpha=18$ which is impossible. Consequently $a_{0}=0$. Again (1), (2) and (3) give $a_{3}=3, a_{4}=a_{2}=0$, and $a_{1}=3$ which imply that $\Gamma=\left(S G_{3},+\right)$.

Next suppose that $\sigma=-$. By Proposition 2.1, $\Gamma$ is a connected unicyclic graph. As in the case $\sigma=+$, since $a_{0}=0$, we have $a_{4}=0$ and $a_{3}=3$. Once more equations (1) and (2) yield $a_{1}=3$ and $a_{2}=0$. Therefore $\Gamma=\left(S G_{3},-\right)$.

Lemma 2.7. Let $\Gamma$ be a signed graph without isolated vertices which is $L$-cospectral with $\Lambda=\left(S G_{n}, \sigma\right)$, where $n \geq 4$. If $\beta=t_{\Gamma}^{-}-t_{\Gamma}^{+}$, then $a_{1}=n+\beta, a_{2}=-3 \beta, a_{3}=n+3 \beta$ and $a_{4}=-\beta$.

Proof. Since $n \geq 4$, we have $t_{\Lambda}^{-}=t_{\Lambda}^{+}=0$. By Theorem $1.5(\mathrm{v})$,

$$
6 \beta+\sum_{i=1}^{4} i^{3} a_{i}=28 n
$$

By (3) and (5) we have,

$$
2 a_{2}+9 a_{3}+24 a_{4}=9 n-3 \beta \text {. }
$$

Also (2) and (3) give

$$
2 a_{2}+6 a_{3}+12 a_{4}=6 n .
$$

Therefore from (6) and (7) we deduce that

$$
a_{3}+4 a_{4}+\beta=n
$$

Now from (4) and (8) we get $a_{3}=n+3 \beta$ and $a_{4}=-\beta$; and from (1) and (2), $a_{1}=n+\beta$ and $a_{2}=-3 \beta$.

Lemma 2.8. A tree with at least a vertex of degree 3 whose vertex degrees are either 1 or 3, has two pendant vertices sharing the same neighbour.

Theorem 2.9. Let $\Gamma$ be a signed graph L-cospectral with $\Lambda=\left(S G_{n},-\right)$ and $n \geq 4$. Then $\Gamma$ is switching isomorphic to $\Lambda$.

Proof. By Lemma 2.1, $\Gamma$ is a connected unicyclic graph. As in the Lemma 2.7, set $\beta=t_{\Gamma}^{-}-t_{\Gamma}^{+}$. If the unique cycle of $\Gamma$ is a triangle, then $\beta=1=-a_{4}$ by Lemma 2.7, which is impossible. Hence $\beta=0$ and therefore $a_{1}=a_{3}=n$ and $a_{2}=a_{4}=0$, again by Lemma 2.7. Let the cycle 
of $\Gamma$ be of length $q$. Since no vertex of $\Gamma$ is of degree 2 , we have $q \leq n$. Now the structure of $\Gamma$ is such that to every vertex $x$ of its cycle a tree is attached so that the degree of $x$ is 3 in $\Gamma$.

These trees have only vertices of degrees 1 and 3. If $q<n$, some of these trees have vertices of degree 3 and Lemma 2.8 says that they have two pendant vertices sharing the same neighbor. Note that such a shared neighbor is not a vertex of the cycle. Hence by Lemma 1.7, $\Gamma$ has 1 as a Laplacian eigenvalue while $\Lambda$ is not so. Therefore $q=n$ and $\Gamma$ is a sun (switching) isomorphic to $\Lambda$.

Theorem 2.10. Let $\Gamma$ be $L$-cospectral with $\Lambda=\left(S G_{n},+\right)$, where $n \geq 5$ is odd. Then $\Lambda$ and $\Gamma$ are switching isomorphic.

Proof. By Theorem 1.5 (ii), $\Lambda$ has a simple zero as a Laplacian eigenvalue and so does $\Gamma$. Therefore $\Gamma$ consists of a balanced component and some unbalanced components.

We have $\left|b_{2 n-1}(\Lambda)\right|=2 n^{2}=\left|b_{2 n-1}(\Gamma)\right|$. Let $c$ be the number of unbalanced components of $\Gamma$. If $c>0$ then 4 divides $\left|b_{2 n-1}(\Gamma)\right|$ by Theorem 1.3, which is a contradiction to the oddness of $n$. Hence $c=0$ and $\Gamma$ is a balanced connected graph. Since the number of edges and vertices of $\Gamma$ are equal, $\Gamma$ is a unicyclic graph. Let the unique cycle of $\Gamma$ be of length $q$. Then $\left|b_{2 n-1}(\Lambda)\right|=2 n^{2}=\left|b_{2 n-1}(\Gamma)\right|=2 n q$, which enforces $q=n$. Since $n \geq 5$, in Lemma 2.7, $\beta=0$. Therefore $a_{1}=a_{3}=n$ and $a_{2}=a_{4}=0$. Since the lengths of the cycles of $\Gamma$ and $\Lambda$ are equal, they are (switching) isomorphic.

Example 2.11. The following graph is L-cospectral with $\Lambda=\left(S G_{4},+\right)$, as an easy calculation with MATLAB R2017a shows. Dashed lines are the negative edges.

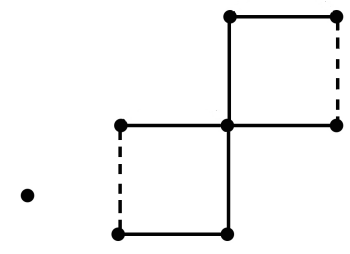

Proposition 2.2. Let $\Lambda=\left(S G_{n},+\right)$ be a signed graph, where $n$ is even. If $n \geq 6$, then $\Lambda$ is $L$-cospectral with $\left(S G_{\frac{n}{2}},+\right) \cup\left(S G_{\frac{n}{2}},-\right)$.

Proof. By Lemma 2.1, Laplacian eigenvalues of $\Lambda$ are $\left(2-\lambda_{i}\right) \pm \sqrt{\left(\lambda_{i}-1\right)^{2}+1}$, where $\lambda_{i}=\cos \frac{2 i}{n} \pi(i=0, \ldots, n-1)$. For even $i$, they are the eigenvalues of $\left(S G_{\frac{n}{2}},+\right)$, and for odd $i$, they are the eigenvalues of $\left(S G_{\frac{n}{2}},-\right)$.

Summing up, we have our main theorem as follows.

Theorem 2.12. The signed sun graph $\Lambda=\left(S G_{n},-\right), n \geq 3$, is determined by its Laplacian spectrum. The signed sun graph $\Lambda=\left(S G_{n},+\right), n \geq 3$, is determined by its Laplacian spectrum if and only if $n$ is odd.

Proof. All ingredients are now ready. Use Theorems 2.6, 2.9, 2.10, Example 2.11 and Proposition 2.2 . 


\section{References}

[1] F. Belardo, P. Petecki, Spectral characterizations of signed lollipop graphs, Linear Algebra Appl., Volume(480):144-167, (2015).

[2] F. Belardo, S. K. Simić, On the Laplacian coefficient of signed graphs, Linear Algebra Appl., Volume(475):94-113, (2015).

[3] R. Boulet, Spectral characterizations of sun graphs and broken sun graphs, Discrete Mathematical Theoretical Computer Science, i., Volume(11)\#2:149-160, (2009).

[4] R. Boulet, B. Jouve, The lollipop graph is determined by its spectrum, Electron. J. Combin., Volume(15):research paper R\#74, (2008).

[5] C. Bu, J. Zhou, H. Li, W. Wang, Spectral characterizations of the corona of a cycle and two isolated vertices, Graphs Combin. Volume(30):1123-1133, (2014).

[6] S. Chaiken, A combinatorial proof of the all minors matrix tree theorem, SIAM J. Algebr. Discrete Methods 3, Volume(3):319-329, (1982).

[7] W. H. Haemers, X.-G. Liu, Y.-P. Zhang, Spectral characterizations of lollipop graphs, Linear Algebra Appl., Volume(428): 2415-2423, (2008).

[8] Y. Hou, J. Li, Y. Pan, On the Laplacian eigenvalues of signed graphs, Linear Multilinear Algebra, Volume(51):21-30, (2003).

[9] M. Mirzakhan, D. Kiani, The sun graph is determined by its signless Laplacian spectrum, Electronic Journal of Linear Algebra, Volume(20):610-620, (2010). 